\title{
Differentiation of pityriasis rubra pilaris from plaque psoriasis by dermoscopy
}

\author{
Abhijeet Kumar Jha ${ }^{1}$, Aimilios Lallas², Sidharth Sonthalia ${ }^{3}$, Deepak Jhakar ${ }^{4}$, \\ Uday K. Udayan ${ }^{1}$, R. K. P. Chaudhary ${ }^{1}$
}

\begin{abstract}
1 Department of Skin \& VD, Patna Medical College and Hospital, Patna, Bihar, India
2 First Department of Dermatology, Aristotle University, Thessaloniki, Greece

3 SKINNOCENCE: The Skin Clinic, Gurgaon, India

4. Department of Dermatology, Deen Dayal Upadhyay Hospital, New Delhi, India
\end{abstract}

Key words: pityriasis rubra pilaris, plaque psoriasis, lichen spinulosus, lichen nitidus, dermoscopy

Citation: Jha AK, Lallas A, Sonthalia S, Jhakar D, Kumar Udayan UK, Chaudhary RKP. Differentiation of pityriasis rubra pilaris from plaque psoriasis by dermoscopy. Dermatol Pract Concept. 2018;8(4):299-302. DOI: https://doi.org/10.5826/dpc.0804a10

Received: February 8, 2018; Accepted: July 3, 2018; Published: October 31, 2018

Copyright: $@ 2018$ Jha AK et al. This is an open-access article distributed under the terms of the Creative Commons Attribution License, which permits unrestricted use, distribution, and reproduction in any medium, provided the original author and source are credited.

Funding: None.

Competing interests: The authors have no conflicts of interest to disclose.

All authors have contributed significantly to this publication.

Corresponding author: Abhijeet Kumar Jha, MD, Assistant Professor, Department of Skin and VD, Patna Medical College, Ashok Rajpath, Patna-800004, Bihar, India. Email: drabhijeetjha@gmail.com

\footnotetext{
ABSTRACT Background: Diagnosing and differentiating pityriasis rubra pilaris (PRP) from other disorders can be a challenging task. Although histopathology remains the gold standard, it may not be feasible at times, especially in children. Being noninvasive, dermoscopy can be of great diagnostic importance in such a scenario. Dermoscopy overcomes the refractive properties of stratum corneum by interface medium or cross-polarization enabling easier visualization of lesions.

Objective: To study the dermoscopic features of PRP and compare them with dermoscopic features of psoriasis.

Patients and Methods: Retrospective observational analysis of dermoscopic characteristics of 28 patients with PRP (16) and psoriasis (12). Polarized dermoscopy at 10x magnification was performed and photographs were captured by Apple iPhone 7. The selection of dermoscopic variables was based on preexisting literature on the dermoscopic patterns of the 2 entities.

Results: The most frequent dermoscopic criteria of PRP were the presence of a central hair (16/16; $100 \%)$, follicular plugs $(11 / 16 ; 68.7 \%)$, and perifollicular yellow/orange halos $(9 / 16 ; 56.2 \%)$. No vascular structures were seen in our cases of PRP. Psoriasis was characterized by regularly distributed dotted vessels $(12 / 12 ; 100 \%)$ and white scales $(8 / 12 ; 66.6 \%)$.

Conclusions: Round-to-oval yellowish areas surrounding a central hair with or without follicular plugs represents the most frequent dermoscopic pattern of PRP.
} 


\section{Introduction}

Pityriasis rubra pilaris (PRP) is a relatively uncommon skin disease, clinically characterized by follicular keratotic plugs, red to orange plaques, and palmoplantar hyperkeratosis. However, in cases with atypical clinical presentation, PRP is sometimes confused with psoriasis, both clinically and histopathologically. In such cases, repeated biopsies may be needed to confirm the diagnosis. The main differential diagnosis of adult-onset PRP is psoriasis.

Dermoscopy has been shown to represent a useful clinical tool for the clinical evaluation of several inflammatory skin diseases and the differential diagnosis among entities with similar clinical manifestations. Available data of the dermoscopic morphology of PRP are scant, whereas evidence on the value of dermoscopy for discriminating PRP from psoriasis is even poorer.

The aim of the present study was to investigate the dermoscopic findings in a series of patients with PRP as compared with those in a control group of patients with psoriasis.

\section{Patients and Methods}

In this retrospective observational study we evaluated the dermoscopic criteria of PRP and psoriasis. Ethics committee approval was obtained. The database of our center was screened for eligible patients. Inclusion criteria included a histopathological diagnosis of PRP or plaque psoriasis (PP) affecting the trunk and/or upper or lower extremities and the availability of a clinical and dermoscopic image of a recently appeared lesion. Patient demographics were recorded and the most recently evolved lesion was examined dermoscopically and histopathologically. Dermoscopy preceded histology, and no treatment was allowed between these examinations. Patients with previous history of treatment were excluded. Dermoscopic examination was performed with a handheld Dermatoscope (DermLite DL 4, 3Gen, San Juan Capistrano, CA) and the images were captured with Apple iPhone 7. Dermoscopic evaluation was retrospectively performed by 2 independent dermoscopists (with more than 10 and 5 years of experience, respectively) who were unaware of the clinical and histopathological diagnosis. The sequence of the dermoscopic images was randomized, using a computer-generated program, and the 2 evaluators were blinded with respect to clinical information and diagnosis. Selection of the dermoscopic variables included in the evaluation process was based on the available published data and personal experience and expertise of the examiners.

The selection of dermoscopic variables was based on previous evidence on the dermoscopic morphology of PRP and psoriasis, as well as our personal observations, and
TABLE 1. Dermoscopic variables

\begin{tabular}{|l|c|c|}
\hline $\begin{array}{c}\text { Dermoscopic Criteria } \\
\text { (Polarized, 10x) }\end{array}$ & $\begin{array}{c}\text { PRP } \\
\text { (n= 16) }\end{array}$ & $\begin{array}{c}\text { Psoriasis } \\
\text { (n= 12) }\end{array}$ \\
\hline Dotted Vessels & 0 & $12(100 \%)$ \\
\hline Linear Vessels & 0 & 0 \\
\hline $\begin{array}{l}\text { Perifollicular Yellow/ } \\
\text { Orange Halos }\end{array}$ & $9(56.2 \%)$ & 0 \\
\hline Follicular Plugs & $11(68.7 \%)$ & 0 \\
\hline Central Hair & $16(100 \%)$ & 0 \\
\hline White Scales & & $8(66 \%)$ \\
\hline
\end{tabular}

included dotted and linear vessels, white scales, follicular plugs, perifollicular yellow/orange halos, and central hair (Table 1). Histological diagnosis of PRP and PP was based on the identification of the characteristic features of each disease under microscopic examination. Analytically, alternating orthokeratosis and parakeratosis in both directions, hypergranulosis, irregular acanthosis, thick suprapapillary plates, and sparse-to-moderate lymphocytic perivascular infiltrate in the dermis, and dilated hair follicles filled with a dense, horny plug were criteria for PRP. In contrast, psoriasiform epidermal hyperplasia accompanied by tortuous, dilated capillaries in the superficial papillary dermis and a perivascular mononuclear cell infiltrate were the criteria for the histopathological diagnosis of PP.

Any disagreement in the assessment of morphological criteria and diagnosis between the 2 dermoscopists or pathologists were resolved by consensus. All evaluators were unaware of patients' clinical details.

\section{Results}

Overall, 28 lesions from 28 patients were included in the study. The histopathological diagnosis was PRP in 16 patients and PP in 12 patients. The male-to-female ratio was 3.5:1 and the mean age was 18.2 years. As shown in Table 1 , the most frequent dermoscopic criteria of PRP were the presence of a central hair $(16 / 16 ; 100 \%)$, follicular plugs $(11 / 16 ; 68.7 \%)$, and perifollicular yellow/orange halos $(9 / 16 ; 56.2 \%)$ (Figures 1 and 2). No vascular structures were seen in our cases of PRP. Psoriasis was characterized by regularly distributed dotted vessels $(12 / 12 ; 100 \%)$ and white scales (8/12; 66.6\%) (Figure 3, Table 1).

\section{Discussion}

Our results suggest that the dermoscopic pattern of PRP consists of yellow/orange halos surrounding follicles with a 


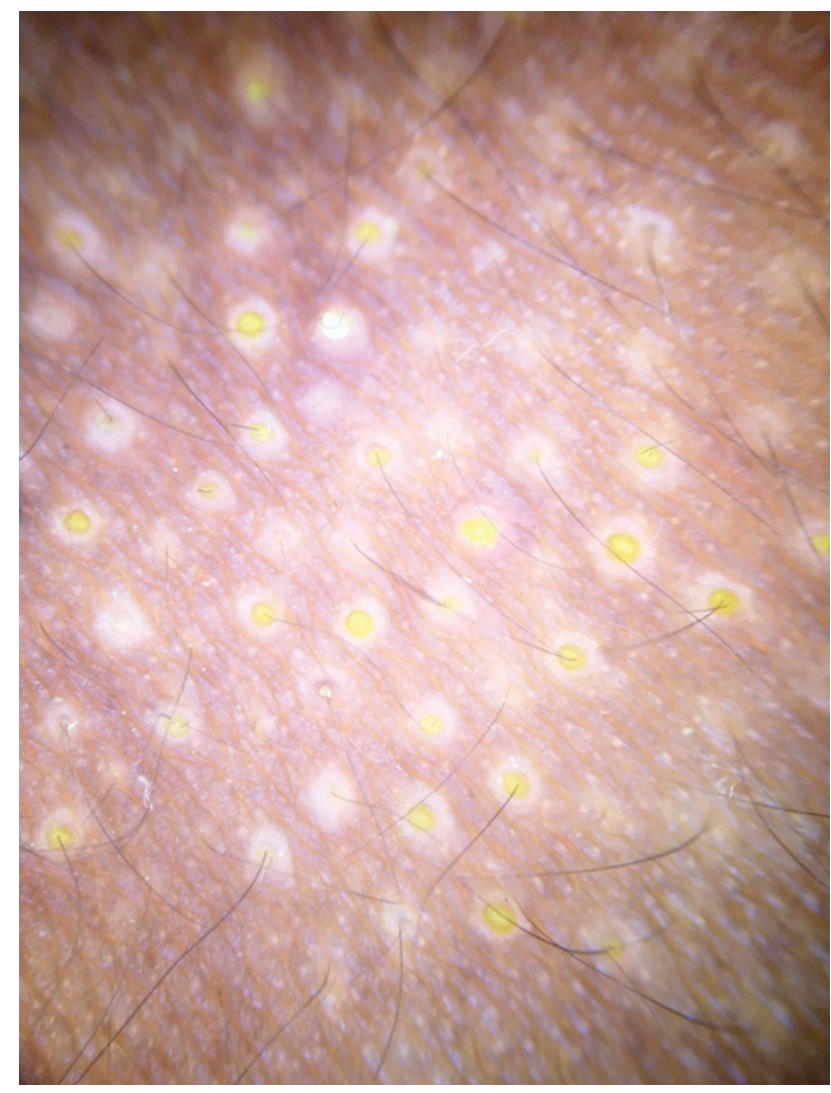

Figure 1. Multiple round-to-oval yellowish areas with keratotic follicular plugs surrounded by erythema and the presence of a central hair (dermoscopy, polarized, 10x). [Copyright: @2018 Jha et al.] central hair and with or without follicular plugs. In contrast, psoriasis is typified by the presence of dotted vessels in a regular distribution and white scales.

Previous reports on dermoscopy of PRP suggested that the disease is dermoscopically characterized by round-to-oval yellowish areas surrounded by vessels of mixed morphology, namely, linear and dotted [1-3]. In addition, central keratin plugs were also described [1]. Our results are partially consistent with previous reports, since we also identified yellowish areas and follicular plugs. Our results suggest that the yellowish areas of PRP are almost always perifollicular. This information was not provided in previous reports. In addition, in our series vessels were not observed. This discrepancy with previous studies might be related to the different skin types of populations, since the increased pigment in the skin of our patients might have impeded the visualization of vascular structures.

Our findings on the features of psoriasis were in perfect agreement with previous reports, suggesting that the disease is dermoscopically typified by regularly distributed dotted vessels and white scales. It is remarkable that although our study and previous studies were conducted in populations of different skin types, the dermoscopic pattern of psoriasis appears

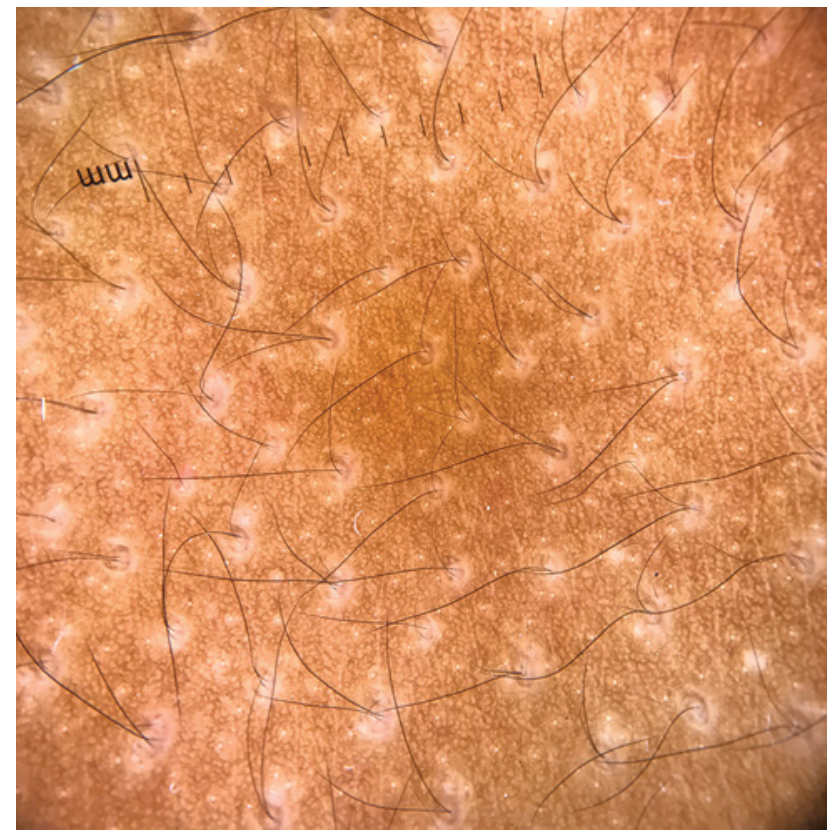

Figure 2. Multiple round-to-oval whitish areas with keratotic follicular plugs surrounded by erythema and the presence of a central hair (dermoscopy, polarized, 10x). [Copyright: @2018 Jha et al.]

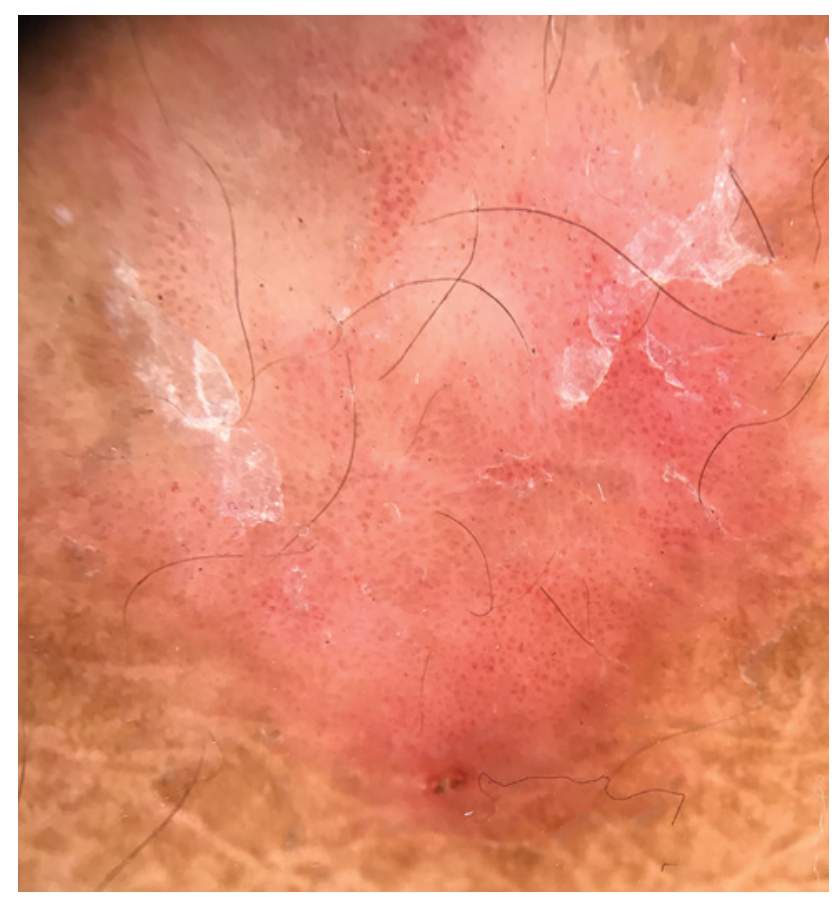

Figure 3. Regular dotted vessels with white scales on a reddish background (dermoscopy, polarized, 10x). [Copyright: @2018 Jha et al.]

to remain identical. It seems that the vascular structures of psoriasis are too prominent to be hidden by the increased pigment of patients with dark skin type.

Overall, our results suggest that dermoscopy might be particularly useful to discriminate between PRP and psoriasis, at least in an Indian population, since the dermoscopic patterns of the 2 entities are clearly different. Of note, yellow color, which was in our study and previous studies 
one of the consistent findings of PRP, has been suggested as a potent negative predictive factor for the diagnosis of psoriasis. A previous study investigated the accuracy of dermoscopic criteria for the differentiation of psoriasis from other inflammatory diseases. According to the latter study, the presence of regularly distributed dotted vessels is very highly suggestive of psoriasis over any other diagnosis. Although the latter study did not include PRP in the control group, our results and previous results on dermoscopy of PRP suggest that the same conclusion is very likely to be valid for the differential diagnosis between psoriasis and PRP. In addition, the latter study suggested yellow color as a potent negative predictive factor for the diagnosis of psoriasis. In the present and previous studies, yellow color was one of the most consistent findings of PRP, supporting further that dermoscopy is useful for the differential diagnosis between PRP and psoriasis.

Previous reports investigated also the dermoscopic findings in peculiar subtypes of PRP. Specifically, erythrodermic PRP was suggested to dermoscopically display orange blotches and islands of nonerythematous (spared) skin displaying reticular vessels $[4,5]$. In contrast, erythrodermic psoriasis is typified by diffusely distributed whitish scales and regularly arranged dotted/glomerular vessels [6].

Keratoderma resulting from PRP has been suggested to display structureless orange areas of different sizes in a patchy distribution along with white scales [4]. Dermoscopy of juvenile PRP also shows multiple whitish keratotic follicular plugs and a yellowish peripheral keratotic ring surrounded by erythema with some linear vessels [7]. Our sample did not include patients with these peculiar forms of PRP, so the latter evidence cannot be assessed by the present study.

\section{Limitations}

The number of patients recruited in the study was small and larger scale studies are required. All patients were Indian and interethnic variability could not be evaluated.

\section{Conclusions}

Round-to-oval yellowish areas surrounding a central hair with or without follicular plugs represents the most frequent dermoscopic pattern of PRP. In contrast, psoriasis is typified by numerous and regularly distributed dotted vessels and white scales. This information might aid clinicians to differentiate atypical cases of PRP from psoriasis.

\section{References}

1. Errichetti E, Stinco G. The practical usefulness of dermoscopy in general dermatology. G Ital Dermatol Venereol. 2015;150(5):533-546.

2. Lallas A, Apalla Z, Karteridou A, Lefaki I. Photoletter to the editor: Dermoscopy for discriminating between pityriasis rubra pilaris and psoriasis. J Dermatol Case Rep. 2013;7(1):20-22.

3. Errichetti E, Stinco G. Dermoscopy as a supportive instrument in the differentiation of the main types of acquired keratoderma due to dermatological disorders. J Eur Acad Dermatol Venereol. 2016;30(12):e229-e231.

4. Errichetti E, Piccirillo A, Stinco G. Dermoscopy as an auxiliary tool in the differentiation of the main types of erythroderma due to dermatological disorders. Int J Dermatol. 2016;55(12):e616-e618.

5. López-Gómez A, Vera-Casaño Á, Gómez-Moyano E, et al. Dermoscopy of circumscribed juvenile pityriasis rubra pilaris. J Am Acad Dermatol. 2015;72(1 suppl):S58-S59.

6. Errichetti E, Stinco G. Dermoscopy in general dermatology: a practical overview. Dermatol Ther (Heidelb). 2016;6(4):471-507.

7. Lallas A, Kyrgidis A, Tzellos TG, et al. Accuracy of dermoscopic criteria for the diagnosis of psoriasis, dermatitis, lichen planus and pityriasis rosea. Br J Dermatol. 2012; 66(6):1198-1205. 